\title{
A Feature Selection for Detection of Non ST Elevation Myocardial Infarction
}

\author{
César Navarro ${ }^{1}$, Mary Jo Kurth², David J McEneaney ${ }^{3}$, James McLaughlin ${ }^{1}$ \\ ${ }^{1}$ Connected Health and Innovation Centre (CHIC) Ulster University, Jordanstown, Northern Ireland \\ ${ }^{2}$ Randox Laboratories Ltd, Crumlin, UK \\ ${ }^{3}$ Craigavon Area Hospital, Craigavon, Northern Ireland
}

\begin{abstract}
A feature selection (FS) process of biomarkers for detecting Acute Myocardial Infarction (AMI) for Non ST Elevation patients (NSTEMI) is presented. FS has been applied by a retrospective analysis of biomarkers - high sensitivity cardiac troponin $T$ (hs-cTnT), heart-type fatty acid-binding protein (H-FABP), creatine kinase-MB (CK-MB), myoglobin, GPBB, CA3 and NTproBNP which are measured at different times from presentation. ECG anomalies at presentation which are key for diagnosis were not considered for FS since they are routinely assessed in the emergency department (ED). Biomarkers measurements and additional data were collected at the ED from patients with chest pain of suspected cardiac origin comprising 478 cases (97 NSTEMI).

hs-cTnT, H-FABP and CK-MB are statistically significant biomarkers to detect AMI according to ROC curve analysis and logistic regressions using data at different time windows. Overall, hs-cTnT as a sole marker is superior for AMI detection. However, H-FABP can be detected earlier and it demonstrates net gains in classification for non-AMI that makes it relevant for AMI rule-out approaches.
\end{abstract}

\section{Introduction}

Chest pain is one of the most common complaints with patients arriving at Emergency Department (ED) with approximately 6 million patients presenting annually in the United States [1]. Of those admitted, only $10 \%$ to $13 \%$ have a confirmed Acute Myocardial Infarction (AMI) [2]. Challenges exist in providing early and accurate diagnosis of AMI in patients admitted to ED with chest pain of suspected cardiac origin. Additionally, many current standard diagnostic tests are not accurate enough to rule-out MI at presentation [3] or under 3 hours in ECG negative patients providing a burden to overcrowded ED. Inappropriate discharge however is associated with a 5-fold increase in mortality and morbidity [4].

AMI is diagnosed by history, clinical examination, electrocardiogram (ECG) and measurement of cardiac biomarkers including high sensitive troponin $\mathrm{T}$ (hscTnT).

This study aimed to explore additional biomarkers that could add more diagnostic capabilities to hs-cTnT (which is the gold standard) in the detection of AMI in patients presented to ED with chest pain of suspected cardiac origin. This retrospective analysis for feature selection takes biomarkers measured at multiple timepoints after presentation apart from the initial ECG to assist in the detection of AMI.

\section{Methods}

\subsection{Recruitment of patients and data}

Consecutive patients (age $>18$ years) with chest pain of presumed ischaemic origin were recruited at Craigavon Area and Daisy Hill hospitals in Northern Ireland between October 2009 and October 2011. Exclusion criteria comprise symptom onset $>12$ hours prior to recruitment, had a terminal malignancy, had received thrombolysis prior to recruitment, were receiving chronic anticoagulant therapy or had been previously recruited into the study. Time of symptom onset was determined following patient interview. The study complies with the Declaration of Helsinki and was approved both by the Office for Research Ethics Northern Ireland and the Southern Health and Social Care Trust Research and Development Department. Written informed consent was obtained from all participating patients.

A 12-lead ECG was obtained on presentation and indicators of anomalies were annotated (abnormal Q wave, ST shift and $\mathrm{T}$ wave inversion). Blood was drawn on presentation (time 0 hour) and at subsequent timepoints if required (1, 2, 3, 6, 12 and 24 hours) for the measurement of biomarkers hs-cTnT, Creatine KinaseMB (CK-MB), Myoglobin, GPBB, Heart-type Fatty Acid-Binding Protein (H-FABP), CAIII, Troponin I (cTnI), Troponin T (cTnT) and NTproBNP.

Diagnosis of AMI was established according to the universal definition of MI at the time [5]. The final diagnosis was adjudicated by two independent clinicians 
who had all the clinical, laboratory and imaging data available.

548 cases were recruited and 70 cases of ST Elevation MI (STEMI) were not included in the analysis since those cases are treated by PCI (in hospitals where it is available) and would not routinely have biomarkers measured. Thus the number of cases was reduced from 548 to 478 including 97 Non ST Elevation MI (NSTEMI).

\subsection{Feature selection}

Measured biomarkers for segregating cases of MI from non-MI were analysed by the area under the receiver operator characteristic curve (AUROC) and logistic regression. Calculations were carried out using $\mathrm{R}$ version 3.1.0 and for AUROC analysis the package "pROC" [6] was used. Level of statistical significance was fixed at 0.05 for comparisons. Values were rounded to four decimal places. The biomarkers exhibiting the highest AUROC with no statistical significant difference were chosen as candidate markers for further exploration. Cases with missing values were excluded and no imputation of the data was carried out.

\section{Results}

\subsection{AUROC}

H-FABP， hs-cTnT， CK-MB， GPBB， CAIII, Myoglobin, c-TnI, NTproBNP and c-TnT were analysed using AUROC at different times from presentation.

According to table 1, hs-cTnT at presentation had the highest AUROC of all the biomarkers but was not statistically different to H-FABP ( $\mathrm{p}=0.0587$ ).

Table 1. AUROC for biomarkers associated to AMI at presentation. Lower and upper CI 95\% and p value compared to hs-cTnT.

\begin{tabular}{llllll}
\hline Biomarker & Area & Std e & Lower & Upper & $\mathrm{p}$ \\
\hline hs-cTnT & 0.9083 & 0.021 & 0.867 & 0.9495 & NA \\
CK-MB & 0.7973 & 0.027 & 0.7443 & 0.8503 & 0.0013 \\
Myoglobin & 0.7297 & 0.0314 & 0.6682 & 0.7911 & 0 \\
GPBB & 0.5767 & 0.0343 & 0.5095 & 0.644 & 0 \\
H-FABP & 0.8483 & 0.0237 & 0.8019 & 0.8947 & 0.0587 \\
CAIII & 0.5231 & 0.0326 & 0.4592 & 0.587 & 0 \\
cTnI & 0.6576 & 0.0266 & 0.6054 & 0.7097 & 0 \\
NTproBNP & 0.6499 & 0.0333 & 0.5847 & 0.7151 & 0 \\
cTnT & 0.8195 & 0.0261 & 0.7682 & 0.8707 & 0.0083 \\
\hline
\end{tabular}

The pattern of superiority for AUCROC of hs-cTnT is repeated at 3 hours (table 2) but it is not statistically different to H-FABP $(\mathrm{p}=0.1239)$.
Table 2. AUROC for biomarkers associated to AMI at 3 hours. Lower and upper CI 95\% and p value compared to hs-cTnT.

\begin{tabular}{lcllll}
\hline \multicolumn{1}{c}{ Biomarker } & Area & Std e & Lower & Upper & $\mathrm{p}$ \\
\hline hs-cTnT & 0.9155 & 0.023 & 0.8705 & 0.9605 & NA \\
CK-MB & 0.8438 & 0.0262 & 0.7926 & 0.8951 & 0.0397 \\
Myoglobin & 0.7142 & 0.0341 & 0.6475 & 0.781 & 0 \\
GPBB & 0.6004 & 0.0341 & 0.5335 & 0.6673 & 0 \\
H-FABP & 0.8676 & 0.021 & 0.8265 & 0.9088 & 0.1239 \\
CAIII & 0.5643 & 0.0343 & 0.497 & 0.6315 & 0 \\
cTnI & 0.7676 & 0.0281 & 0.7126 & 0.8226 & 0 \\
cTnT & 0.4737 & 0.0301 & 0.4147 & 0.5327 & 0 \\
\hline
\end{tabular}

\subsection{Logistic regression}

Logistic regressions were used to show the predictive capabilities of combinations of biomarkers collected at different timepoints. hs-cTnT, H-FABP and CK-MB were included as predictors of AMI in two models for data collected up to 1 hour and 3 hours.

A model is presented in table 3 with data collected up to 1 hour from presentation ( 0 hour) that used biomarkers from 335 patients, 67 NSTEMIs and without missing values for the biomarkers involved. A corresponding label for the timepoint was added to the name of the biomarker for naming the variables.

Table 3. Logistic regression model with data collected up to 1 hour from presentation. 335 patients, 67 NSTEMIs.

\begin{tabular}{lllll}
\hline & estimate & Std e & $\mathrm{t}$-val & $\mathrm{p}$ \\
\hline Intercept & -2.5318 & 0.2754 & -9.193 & 0 \\
hs-cTnT_0 & 0.0164 & 0.00851 & 1.928 & 0.0539 \\
hs-cTnT_1 & 0.00071 & 0.006 & 0.118 & 0.9062 \\
H-FABP_0 & -0.4546 & 0.1225 & -3.71 & 0.0002 \\
H-FABP_1 & 0.6614 & 0.1678 & 3.941 & 0 \\
CK-MB_0 & 0.0678 & 0.2177 & 0.312 & 0.7554 \\
CK-MB_1 & -0.1862 & 0.1724 & -1.08 & 0.28 \\
\hline
\end{tabular}

F critical $5 \%=2.13$, null deviance (total): 335.27 on 334 degrees of freedom (df) and residual deviance: 231.22 on $328 \mathrm{df}$. Model residual: 104.05 on $6 \mathrm{df}$, $\mathrm{F}(6,328)=24.6$, then the null hypothesis Ho of all coefficients equal to zero is rejected. It also can be concluded that coefficients for H-FABP at presentation and at 1 hour are significantly different to zero, suggesting this biomarker is a significant predictor of MI at presentation and 1 hour in the logistic regression model.

Additionally, a model is shown with data collected up to 3 hours from presentation, using biomarkers from 272 patients (without missing values), 55 NSTEMIs, for timepoints at presentation (0 hour), 1 hour, 2 hour and 3 hour (table 4). 
Table 4. Logistic regression model with data collected up to 3 hours from presentation. 272 patients, 55 NSTEMIs.

\begin{tabular}{lllll}
\hline & estimate & Std e & $\mathrm{t}$-val & $\mathrm{p}$ \\
\hline Intercept & -3.6085 & 0.5031 & -7.173 & 0 \\
hs-cTnT_0 & 0.015 & 0.0288 & 0.522 & 0.602 \\
hs-cTnT_1 & -0.0527 & 0.0396 & -1.328 & 0.1841 \\
hs-cTnT_2 & -0.0377 & 0.048 & -0.786 & 0.432 \\
hs-cTnT_3 & 0.1142 & 0.0432 & 2.646 & 0.0081 \\
H-FABP_0 & -0.8702 & 0.2753 & -3.161 & 0.0016 \\
H-FABP_1 & 0.173 & 0.4364 & 0.396 & 0.6919 \\
H-FABP_2 & 0.4071 & 0.5006 & 0.813 & 0.416 \\
H-FABP_3 & 0.3553 & 0.3128 & 1.136 & 0.2559 \\
CK-MB_0 & 0.0608 & 0.5324 & 0.114 & 0.9091 \\
CK-MB_1 & -1.1973 & 0.8542 & -1.402 & 0.161 \\
CK-MB_2 & 2.6305 & 0.875 & 3.006 & 0.0026 \\
CK-MB_3 & -1.4567 & 0.7446 & -1.956 & 0.0504 \\
\hline
\end{tabular}

F critical $5 \%=1.79$, null deviance (total): 273.87 on $271 \mathrm{df}$ and residual deviance of 123.14 on $259 \mathrm{df}$. Model residual: 150.73 on $12 \mathrm{df}, \mathrm{F}(12,259)=26.42$, then reject Ho. Individually, coefficients for H-FABP at presentation, CK-MB at 2 hour and hsTnT at 3 hours are significantly different to zero, suggesting these biomarkers are significant predictors of MI in this logistic regression model.

\subsection{Peaking times for AMI}

Figure 1 shows normalized averages for hs-cTnT, HFABP and CK-MB for the timepoints considered. The steady values below 0.2 correspond for averages of non$\mathrm{MI}$ and are displayed for reference.

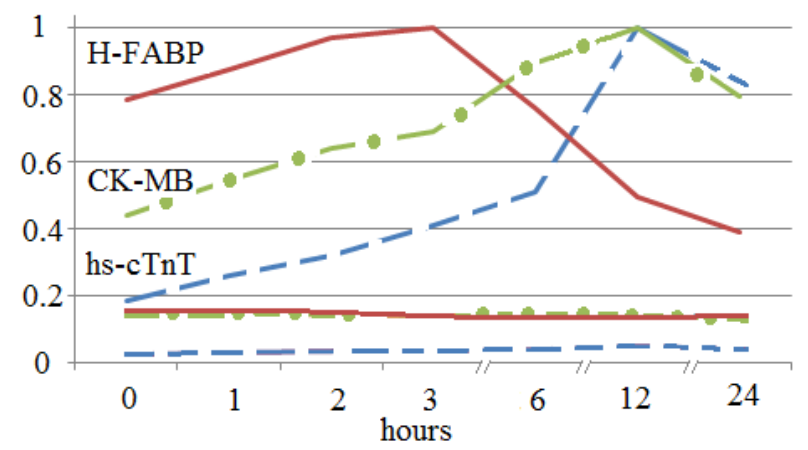

Figure 1. Normalized averages for most relevant biomarkers showing different peaking times for AMI. "0” hour is time of presentation.

\subsection{Comparison of algorithms}

Algorithms to detect MI based on biomarkers at different thresholds were compared using the Pencina's
Net Reclassification Improvement (NRI) [7]. The initial focus was towards algorithms at presentation. H-FABP and hs-cTnT are preferred over CK-MB according to the AUROC and logistic regression models. Some comparisons to the gold standard hs-cTnT $>=14 \mathrm{ng} / \mathrm{l}$ are shown in Table 5. The threshold of 14ng/l is the 99th percentile widely used for AMI detection and it is used here as a reference. For instance, according to the first row in table 5 , an algorithm based on $\mathrm{H}-\mathrm{FABP} \geq 1.5 \mu \mathrm{g} / \mathrm{l}$ to detect $\mathrm{MI}$ at presentation is more sensitive than one based on hs-cTnT $\geq 14 \mathrm{ng} / \mathrm{l}$ (net gain with MI of 0.0462 ) but less specific (negative net gain with non-MI of -0.3765), and the probability of $|\mathrm{NRI}|$ (absolute value of the sum of the two net gains) higher than $|-0.3303|$ is low $(p<0.00005)$ if the hypothesis of NRI zero is assumed. Thus it can be concluded that these two algorithms are statistically different in terms of NRI and the negative value shows no overall improvement of the algorithm. However it is not the case when comparing $\mathrm{H}-\mathrm{FABP} \geq 3.8 \mu \mathrm{g} / \mathrm{l}$ against $\mathrm{hsTnT} \geq 14 \mathrm{ng} / \mathrm{l}(\mathrm{p}=0.1073)$ (third row in table 5). The cutoff of $3.8 \mu \mathrm{g} / \mathrm{l}$ was used to show no difference.

Table 5. Pencina's NRI comparing algorithms with hs$\mathrm{cTnT} \geq 14 \mathrm{ng} / 1$ at presentation to detect MI. 360 patients were included in the analysis without missing values and 72 NSTEMIs. Concentrations are in $\mathrm{ng} / \mathrm{l}$ and in $\mu \mathrm{g} / \mathrm{l}$ for hs-cTnT and H-FABP respectively.

\begin{tabular}{lllll}
\hline Algorithm & Net MI & $\begin{array}{l}\text { Net } \\
\text { non-MI }\end{array}$ & NRI & $\mathrm{p}$ \\
\hline $\mathrm{H}-\mathrm{FABP}>=1.5$ & 0.0462 & -0.3765 & -0.3303 & 0 \\
$\mathrm{H}-\mathrm{FABP}>=2.5$ & -0.1231 & -0.0196 & -0.1427 & 0.0193 \\
$\mathrm{H}-\mathrm{FABP}>=3.8$ & -0.2462 & 0.1373 & -0.1089 & 0.1073 \\
$\mathrm{H}-\mathrm{FABP}>=5.3$ & -0.3846 & 0.2078 & -0.1768 & 0.0328 \\
$\mathrm{H}-\mathrm{FABP}>=7$ & -0.4462 & 0.2235 & -0.2226 & 0.0119 \\
\hline hs-cTnT $>=14$ OR & & & & \\
$\mathrm{H}-\mathrm{FABP}>=2.5$ & 0.0154 & -0.0941 & -0.0787 & 0.0014 \\
hs-cTnT $>=14$ OR & & & & \\
$\mathrm{H}-\mathrm{FABP}>=3.8$ & 0 & -0.0314 & -0.0314 & 0.0047 \\
hs-cTnT $>=14$ OR & & & & \\
$\mathrm{H}-\mathrm{FABP}>=5.3$ & 0 & -0.0157 & -0.0157 & 0.0455 \\
\hline $\begin{array}{l}\text { hs-cTnT }>=14 \\
\text { AND H-FABP }>=1\end{array}$ & 0 & & & \\
hs-cTnT $>=14$ & & 0.0039 & 0.0039 & 0.3173 \\
$\begin{array}{l}\text { AND H-FABP }>=1.5 \\
\text { hs-cTnT }>=14\end{array}$ & -0.0154 & 0.0314 & 0.0160 & 0.3992 \\
\begin{tabular}{l} 
AND H-FABP $>=2.5$ \\
\hline
\end{tabular} & -0.1538 & 0.1020 & -0.0519 & 0.3239 \\
\hline
\end{tabular}

\section{Discussion}

Cardiac troponin is currently the main biomarker assisting in the detection of AMI. With the dataset used (focused on NSTEMI only) the analysis demonstrated that hs-cTnT consistently had a higher AUROC than the other biomarkers but the statistical difference to H-FABP 
could not be stablished at presentation $(\mathrm{p}=0.0587)$ (table 1 ) and at 3 hours after presentation $(p=0.1239)$ (table 2$)$.

H-FABP was also a strong predictor of AMI according to logistic regression models especially at 0 and 1hour (tables 3 and 4). In those models H-FABP was combined with hs-cTnT and CK-MB.

$\mathrm{H}-\mathrm{FABP}$, measured at presentation and acting as a sole biomarker at different thresholds, did not outperform hscTnT $>=14 \mathrm{ng} / \mathrm{l}$ for detecting $\mathrm{MI}$ as shown in table 5. In this comparison it is worth considering that hs-cTnT at $14 \mathrm{ng} / \mathrm{l}$ is used to aid in the diagnosis itself. At best, $\mathrm{H}$ $\mathrm{FABP}>=3.8 \mu \mathrm{g} / \mathrm{l}$ used to diagnose $\mathrm{MI}$ showed no statistical difference to hs-cTnT at $14 \mathrm{ng} / \mathrm{l}$ (row 3). There were other thresholds tested for H-FABP and some of them are shown in table 5 and none of them exhibit $\mathrm{NRI}>0$ when compared to hs-cTnT at $14 \mathrm{ng} / \mathrm{l}$.

Nevertheless, the comparisons of algorithms in table 5 show net gains when a diagnosis of non-MI is made when $\mathrm{H}-\mathrm{FABP}$ at thresholds of 3.8, 5.3 and $7 \mu \mathrm{g} / \mathrm{l}$ are compared to hs-cTnT $>=14 \mathrm{ng} / \mathrm{l}$ (rows 3,4 and 5). However, NRI $<0$ for those algorithms at presentation since the net gains when non-MI are surpassed by net gains in detecting MI.

Additionally in table 5 there are comparisons for some of the possible combinations of H-FABP and hs-cTnT. There were cases of NRI $>0$ when compared to hs$\mathrm{cTnT} \geq 14 \mathrm{ng} / \mathrm{l}$ but without statistical significance. It seems that by enforcing the two markers over a threshold (combinations AND instead of OR) it is possible to get $\mathrm{NRI}>0$ with net gains without MI (rows 9 and 10 in table 5). There is still ongoing research in order to stablish how combining H-FABP with hs-cTnT at different thresholds would help to improve on MI detection.

$\mathrm{H}$-FABP is present in high concentrations in the myocardium 30 minutes after an ischemic episode [8]. Additionally, its relative tissue specificity [9] makes it a suitable biomarker to be investigated. Following $\mathrm{H}$ FABP's advantage of early expression (Figure 1) and net gains in non-MI as a sole marker and in combinations with hs-cTnT, we aimed to combine this biomarker with hs-cTnT and ECG anomalies as a rule-out approach, especially under 3 hours of presentation in line with Body et al. [10]. They demonstrated a model of eight variables including hs-cTnT, H-FABP, an indication of ischaemia from ECG and the outcomes of a clinical assessment. In future work we aim to simplify Body's approach using fewer variables and concentrating uniquely in NSTEMIs. Additionally we aim to use the time for onset of symptoms, which is subjective but could be a very important piece of information.

It is important to note the limitation of the study which includes the use of a relatively small dataset.

\section{Conclusions}

H-FABP has shown promise among other biomarkers investigated to pair with hs-cTnT according to AUROC and logistic regressions. It does not outperform hs-cTnT for the detection of AMI in the dataset investigated. However, its earlier detection in blood following an ischaemic event, and net gains in classification when a diagnosis of non-MI is made, suggest its use as a rule-out test for suspected AMI patients. If effective, this would allow risk stratification and a more efficient use of resources to the health care system.

\section{Acknowledgements}

The authors thank INVEST NI, the funders of this research.

\section{References}

[1] Middleton K, Hing E, Xu J. National Hospital Ambulatory Medical Care Survey: 2005 outpatient department summary. Adv Data. 2007;(389):1-34.

[2] Pope JH, Aufderheide TP, Ruthazer R, Woolard RH, Feldman JA, Beshansky JR, Griffith JL, Selker HP. Missed diagnoses of acute cardiac ischemia in the emergency department. N Engl J Med. 2000;342(16):1163-1170.

[3] Goldman L, Kirtane AJ. Triage of Patients with Acute Chest Pain and Possible Cardiac Ischemia: The Elusive Search for Diagnostic Perfection. Ann Intern Med. 2003;139(12):987-995.

[4] Collinson PO, Boa FG, Gaze DC. Measurement of cardiac troponins. Ann Clin Biochem 2001;38(5):423-449.

[5] Thygesen K, Alpert JS, White HD. Universal definition of myocardial infarction, European Heart Journal. 2007;28(20):2525-2538.

[6] Robin X, Turck N, Hainard A, Tiberti N, Lisacek F, Sanchez J-C, Müller M. pROC: an open-source package for $\mathrm{R}$ and $\mathrm{S}+$ to analyze and compare ROC curves. BMC Bioinformatics 2011;12:77.

[7] Pencina MJ, D’Agostino RB Sr, D’Agostino RB Jr, Vasan RS. Evaluating the added predictive ability of a new marker: From area under the ROC curve to reclassification and beyond. Stat Med 2008;27:157-172.

[8] Kleine AH, Glatz JFC, Van Nieuwenhoven FA, Van der Vusse GJ. Release of heart fatty acid-binding protein into plasma after acute myocardial infarction in man. Mol Cell Biochem. 1992;116:155-162.

[9] Alhadi HA, Fox KA. Do we need additional markers of myocyte necrosis: the potential value of heart fatty-acidbinding protein. QJM 2004;97(4):187-198.

[10] Body R, Carley S, McDowell G, Pemberton P, Burrows G, Cook G, Lewis PS, Smith A, Mackway-Jones K. The Manchester Acute Coronary Syndromes (MACS) decision rule for suspected cardiac chest pain: derivation and external validation. Heart 2014;100(18):1462-1468.

Address for correspondence.

César Navarro

Ulster University, NIBEC, Shore Road, Newtownabbey, Co. Antrim BT37 OQB, Northern Ireland.

c.navarro@ulster.ac.uk 